\title{
A spectrographic study of the symbiotic system Hen $1761^{\star}$
}

\author{
E. Brandi ${ }^{1,2}$, R. Barbá ${ }^{1.3}$, L.G. García ${ }^{1}$, and N. Beltrán ${ }^{1}$ \\ 1 Facultad de Ciencias Astronómicas y Geofísicas, Universidad Nacional de La Plata, 1900 La Plata, Argentina \\ e-mails: ebrandi@fcaglp.fcaglp.unlp.edu.ar; rbarba@fcaglp.fcaglp.unlp.edu.ar; lia@fcaglp.fcaglp.unlp.edu.ar \\ 2 Member of the Carrera del Investigador Científico, Comisión de Investigaciones Científicas de la Provincia de Buenos Aires \\ (CIC), Argentina \\ 3 Member of the Carrera del Investigador Científico, Consejo Nacional de Investigaciones Científicas y Técnicas (CONICET), \\ Argentina
}

Received August 14, 1997; accepted April 24, 1998

\begin{abstract}
This work presents the results of a spectroscopic study in the optical and near infrared region of the symbiotic star Hen 1761. Relative fluxes and radial velocities of emission and absorption lines are obtained on the basis of low, intermediate and high resolution spectra. Our observations show that this scarcely studied object underwent very important spectroscopic variations in the interval 1990-1995. The observed variations are quite similar to those of symbiotic stars where accretion events on a hot dwarf are responsible for an eruptive behavior. Some of the physical parameters of the system are derived.
\end{abstract}

Key words: stars: emission-lines — symbiotic stars stars: variable stars — stars: individual: Hen 1761

\section{Introduction}

Hen $1761\left[\alpha(2000.0)=19^{\mathrm{h}} 42^{\mathrm{m}} 25.9 ; \delta(2000.0)=-68^{\circ}\right.$ $\left.07^{\prime} 41^{\prime \prime}\right]$ is a symbiotic star about which several papers have been published although the knowledge of this system is still very poor indeed. The star was mentioned for the first time by Mayall (1951), as a new peculiar object and Thackeray (1954) classified it as a symbiotic star. He detected emission lines of Hi, He I, He II $\lambda 4686$, C II, C III, $\mathrm{N}$ III, [O III]; the presence of Fe II being uncertain. The continuum, stronger in the red, showed moderate $\mathrm{TiO}$ molecular bands but the presence of atomic absorption lines was

Send offprint requests to: E. Brandi

* Based on observations taken at Complejo Astronómico El Leoncito (CASLEO), operated under an agreement between the Consejo Nacional de Investigaciones Científicas y Técnicas de la República Argentina, the Secretaría de Ciencia y Tecnología de la Nación and the National Universities of La Plata, Córdoba and San Juan. very doubtful. According to Glass \& Webster (1973), the infra-red energy distribution of Hen 1761 corresponded to that of an M star. Allen (1980), classified the late-type component as M3 on the basis of infra-red spectroscopy at $2 \mu \mathrm{m}$ and a distance of $1.4 \mathrm{kpc}$ was estimated. The first published optical spectra of Hen 1761 was shown by Allen (1984) in his Catalogue of Symbiotic Stars. Allen (1982) discards the presence of circumstellar dust because of the colour indices $J-K$ and $H-K$ and considers the object as a S-type symbiotic. Hen 1761 was found to have undergone very important photometric (Munari et al. 1992) and spectroscopic (van Winckel et al. 1993) variations. Since Thackeray's report, written more than forty years ago, the above mentioned authors and Wright \& Allen (1978); Lund \& Tuvikene (1987); Kenyon (1986); Kenyon et al. (1988); Schulte-Ladbeck et al. (1990) and Whitelock \& Munari (1992), have included Hen 1761 together with other symbiotic stars in different researches but the present paper is devoted in particular to this object with the aim of presenting and discussing recent spectroscopic data.

\section{Observations}

The spectroscopic observations were carried out with the $2.15 \mathrm{~m}$ telescope at the Complejo Astronómico El Leoncito, CASLEO (San Juan, Argentina). Medium-low resolution spectrograms were obtained with a Boller \& Chivens spectrograph using a photon-counting Reticon, called Z-Machine, during 1990 and 1991 and a Thompson CCD $(500 \times 592$ pixels $)$ in 1992 . Since 1994 high resolution spectrograms were obtained with a REOSC echelle spectrograph using a Tek CCD $(1024 \times 1024$ pixels $)$. A log of the observations is shown in Table 1 .

Details about the Z-Machine observations and data reduction procedures were given by Barbá et al. (1992). 
Table 1. Log of the spectroscopic observations of Hen 1761

\begin{tabular}{lcccc}
\hline Date & $\begin{array}{c}\mathrm{JD}_{\odot} \\
244+\end{array}$ & Resolution & $\begin{array}{c}\text { Range } \\
(\AA)\end{array}$ & $\begin{array}{c}T_{\exp } \\
(\mathrm{s})\end{array}$ \\
\hline $6 / 7 / 90$ & 8078.8 & 2700 & $5650-7000$ & 600 \\
$9 / 8 / 90$ & 8112.8 & 4100 & $4400-5062$ & 840 \\
$11 / 8 / 90$ & 8114.8 & 2700 & $5830-7100$ & 360 \\
$4 / 11 / 90$ & 8199.6 & 2700 & $5830-7100$ & 480 \\
$4 / 11 / 90$ & 8199.6 & 2700 & $5830-7100$ & 240 \\
$9 / 11 / 90$ & 8204.6 & 4100 & $4400-5062$ & 480 \\
$7 / 4 / 91$ & 8353.9 & 4100 & $4400-5062$ & 720 \\
$8 / 7 / 92$ & 8811.8 & 3400 & $4725-5080$ & 1200 \\
$9 / 7 / 92$ & 8812.7 & 2300 & $6340-7080$ & 300 \\
$8 / 8 / 92$ & 8842.7 & 2600 & $8300-9000$ & 600 \\
$9 / 8 / 92$ & 8843.7 & 2100 & $5730-6475$ & 300 \\
$9 / 8 / 92$ & 8843.7 & 2900 & $6450-7200$ & 240 \\
$9 / 8 / 92$ & 8843.7 & 2900 & $8300-9000$ & 300 \\
$9 / 8 / 92$ & 8843.8 & 2900 & $7680-8400$ & 240 \\
$9 / 8 / 92$ & 8843.8 & 2600 & $7130-7800$ & 240 \\
$14 / 8 / 92$ & 8848.8 & 800 & $3750-5170$ & 300 \\
$26 / 10 / 94$ & 9651.5 & 15000 & $3500-5900$ & 1200 \\
$26 / 10 / 94$ & 9651.6 & 15000 & $3500-5900$ & 1200 \\
$27 / 10 / 94$ & 9652.5 & 15000 & $4900-8150$ & 1800 \\
$28 / 10 / 94$ & 9653.5 & 15000 & $4900-8150$ & 1200 \\
$15 / 8 / 95$ & 9944.7 & 15000 & $4200-7200$ & 2400 \\
$15 / 8 / 95$ & 9944.8 & 15000 & $4200-7200$ & 2400 \\
\hline
\end{tabular}

The CCD data were reduced with standard $\operatorname{IRAF}^{1}$ packages, CCDRED and ONEDSPEC and all the spectra were measured using the SPLOT task within IRAF.

To obtain the flux calibration, standard stars from Stone \& Baldwin's (1983) and Baldwin \& Stone's (1984) lists, were observed each night. An analysis of the flux calibration errors, by flux comparisons of the standard stars, shows that they may be estimated at about $15 \%$ for the Z-Machine images, $5 \%$ for the Thomson CCD images and $20 \%$ in the central part of each order for the REOSC echelle images. However, it must be kept in mind that true flux errors are largely dependent on the tracing of the continuum and the emission line strengths.

\section{Analysis}

\subsection{Spectral variations}

In the spectra of Hen 1761 we confirm the presence of emission lines of $\mathrm{HI}$, He I, He II, C III, N III, [O III] and Fe II and detect O I, Mg II, Ti II, [Fe II], [S II], [Ne III], [Fe IV], [Fe VI], $[\mathrm{Fe}$ VII] and $[\mathrm{CaV}]$. Some members of the Paschen Series are also present as very weak emission lines. A Balmer jump in emission is observed in the images of October 1994. The Figs. 1a and b show two spectral regions between $\lambda \lambda 3750-5170 \AA$ and $\lambda \lambda 6200-7200 \AA$ with the

\footnotetext{
${ }^{1}$ IRAF is distributed by the National Optical Astronomy Observatories, which is operated by the association of Universities for Research in Astronomy, INC., under contract to the National Science Foundation.
}
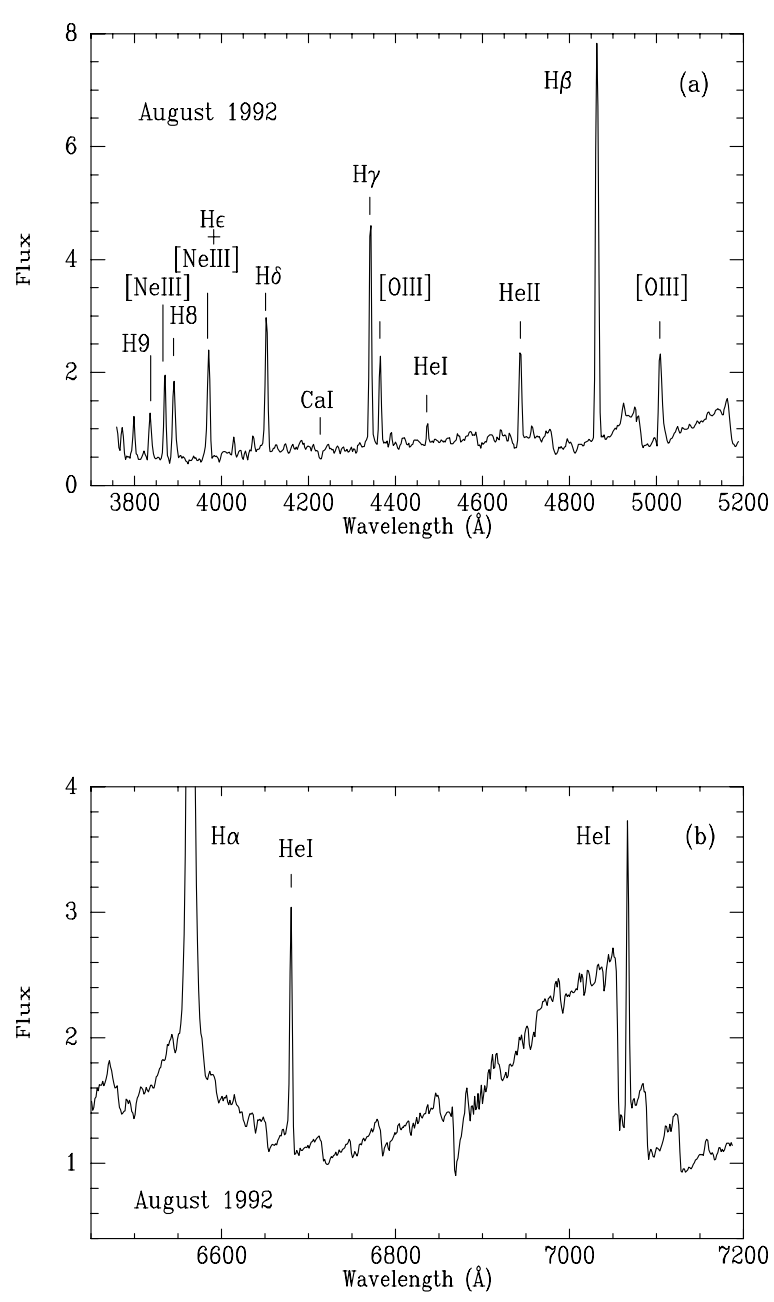

Fig. 1. Low resolution optical spectra for Hen 1761. Spectral regions a) between $\lambda \lambda 3750-5200 \AA$ and b) $\lambda \lambda 6200-7200 \AA$. Numerous $\mathrm{TiO}$ molecular bands are present in the red region. The Raman scattered O VI emission lines at $\lambda 6825 \AA$ and $\lambda 7082 \AA$ are absent. Fluxes are indicated in unit of $10^{-13} \mathrm{erg}$ $\mathrm{cm}^{-2} \mathrm{~s}^{-1} \AA^{-1}$

fluxes indicated in units of $10^{-13} \mathrm{erg} \mathrm{cm}^{-2} \mathrm{~s}^{-1} \AA^{-1}$. As seen in Fig. 1a, numerous $\mathrm{TiO}$ molecular bands are present in the red region and the Raman scattered O VI emission lines at $\lambda 6825 \AA$ and $\lambda 7082 \AA$ (Schmid 1989) are not detected in Hen 1761. The flux ratios of the most important emission lines for the different observing runs, scaled with respect to $\mathrm{H} \beta=100$, are given in Table 2 . The estimated error in the determination of the integrated emission line fluxes is in the order of $\pm 10 \%$ for strong emission lines and $\pm 25 \%$ for weaker lines. The fluxes are not corrected for reddening.

We can see in Table 2 that during 1990-95 Hen 1761 shows variations, in the line intensities of HeI, He II, N III and [O III] in the same sense, reaching in general their minimal values in April 1991, and then increasing until 
Table 2. Line fluxes in the spectrum of Hen 1761 scaled to $\mathrm{H} \beta=100$

\begin{tabular}{|c|c|c|c|c|c|c|c|c|}
\hline \multirow[t]{2}{*}{ Ion } & \multirow{2}{*}{$\begin{array}{c}\lambda \\
(\AA)\end{array}$} & \multicolumn{3}{|c|}{ Z-Machine } & \multicolumn{2}{|c|}{ CCD $500 \times 592$} & \multicolumn{2}{|c|}{ CCD $1024 \times 1024$} \\
\hline & & $8 / 90$ & $11 / 90$ & $4 / 91$ & $7 / 92$ & $8 / 92$ & $10 / 94$ & $8 / 95$ \\
\hline \multirow[t]{5}{*}{ HeI } & 4471 & 4 & 3 & 3 & - & 3 & 2 & 7 \\
\hline & 5015 & 6 & 7 & 3 & 3 & bl & 2 & 8 \\
\hline & 5876 & - & - & - & - & 21 & 15 & 35 \\
\hline & 6678 & - & - & - & - & 15 & 10 & 41 \\
\hline & 7065 & - & - & - & - & 21 & 14 & 59 \\
\hline HeII & 4685 & 19 & 19 & 8 & - & 20 & 23 & 64 \\
\hline NiII & 4634 & 3 & 1 & 1 & - & $\mathrm{w}$ & 1 & 3 \\
\hline NiII & 4640 & 4 & 4 & 2 & - & $\mathrm{w}$ & 3 & 5 \\
\hline \multirow[t]{3}{*}{ [OIII] } & 4363 & - & - & - & - & 15 & 13 & 20 \\
\hline & 4959 & 11 & 6 & 4 & 13 & 10 & 9 & 14 \\
\hline & 5007 & 33 & 21 & 11 & 38 & 26 & 27 & 49 \\
\hline$[\mathrm{Cav}]$ & 5309 & nd & nd & nd & nd & nd & 2 & 8 \\
\hline \multirow[t]{2}{*}{ FeII } & 4629 & 3 & 3 & 2 & - & nd & 1 & $\mathrm{w}$ \\
\hline & 5018 & 5 & 4 & 4 & 1 & $\mathrm{bl}$ & 1 & $\mathrm{w}$ \\
\hline \multirow[t]{3}{*}[\mathrm{FeVI}]{} & 4967 & nd & nd & nd & nd & nd & $\mathrm{w}$ & 3 \\
\hline & 4972 & nd & nd & nd & nd & nd & $\mathrm{w}$ & 3 \\
\hline & 5176 & nd & nd & nd & nd & nd & 2 & 8 \\
\hline \multirow[t]{2}{*}{ [FeviI] } & 5721 & nd & nd & nd & nd & nd & nd & 3 \\
\hline & 6087 & nd & nd & nd & nd & nd & nd & 5 \\
\hline $\mathrm{H} \beta$ flux $^{(*)}$ & & 2.51 & 3.52 & 5.07 & 2.90 & 5.60 & 5.24 & 1.59 \\
\hline
\end{tabular}

Notes:

- : the line is either outside of the observed spectral range or the image does not include $\mathrm{H} \beta$. nd: undetected line

w: very weak line.

bl: blended line.

$(*)$ : The mean absolute fluxes in units of $10^{-12} \mathrm{erg} \mathrm{cm}^{-2} \mathrm{~s}^{-1}$.

August 1995. He II $\lambda 4686 \AA$ and the forbidden line [O III] $\lambda 5007 \AA$ display the most significant intensity variations, but the He I and N III intensities change with a much lower amplitude (see Fig. 2a). In contrast, the Fe II line emissions show a systematic decrease of intensities until 1995. The Fig. 3a shows a spectral region where the variations in the He I, [O III] and Fe II lines are displayed.

Van Winckel et al. (1993) already found dramatic changes in the [O III] $\lambda 5007 \AA$ and Fe II $\lambda 5018 \AA$ lines in time scales of several months. In our spectra $\mathrm{H} \alpha$ is slightly asymmetric on the blue side and a weak central absorption is more noticeable in August 1995. Otherwise, the shape of the $\mathrm{H} \beta$ profile undergo important changes along the time (see Fig. 4). In August 1990 the profile has a symmetrical appearance but a double-peaked profile can be observed in the image of November 1990, being the radial velocity of the central absorption of $49 \mathrm{~km} \mathrm{~s}^{-1}$. H $\beta$ becomes a single emission with an asymmetry on the blue wing, few months later, in April 1991. An incipient central absorption is observed in August 1995.

In the blue region of the spectra the Ti II lines are in absorption in August 1990, then they practically disappear in November 1990, and in April 1991, are visible as weak emissions. Later, up to the last observations, these lines are present as absorptions. Figure $3 \mathrm{~b}$ shows the dramatic variations when the absorptions are filled by emissions.

In 1994 double peak profiles of [Fe VI] $\lambda 4967 \AA \lambda 4972 \AA$ and $\lambda 5176 \AA$ as well as [Ca v] $\lambda 5309 \AA$ appear in the spectrum. Then, in 1995 these lines become stronger and other forbidden lines of higher ionization, like [Fe VII] $\lambda 5721 \AA$ and $\lambda 6087 \AA$ can be detected.

In order to establish the behavior of the continuum as function of a time, we have chosen to measure fluxes in $50 \AA$ bands centered at 4895 and $6975 \AA$ since these spectral ranges are free of important emission lines. The respective fluxes have been converted into a color index $C I=m_{4895}-m_{6975}=-2.5 \log F_{4895} / F_{6975}$ listed in Table 4. In Fig. $2 \mathrm{~b}$ we can see that the system is much bluer during August-November 1990 and later, in July 1992 the red continuum becomes stronger.

From 1990 to 1992 the spectra are dominated by many narrow absorption lines which were identified more frequently as FeI and TiI. Moreover, other absorption lines of neutral and once ionized metals, e.g. Ca I $\lambda 4227 \AA \mathrm{Ni}$, Cri, Na I, O I, Vi, Ca II and Ni II are also present. 

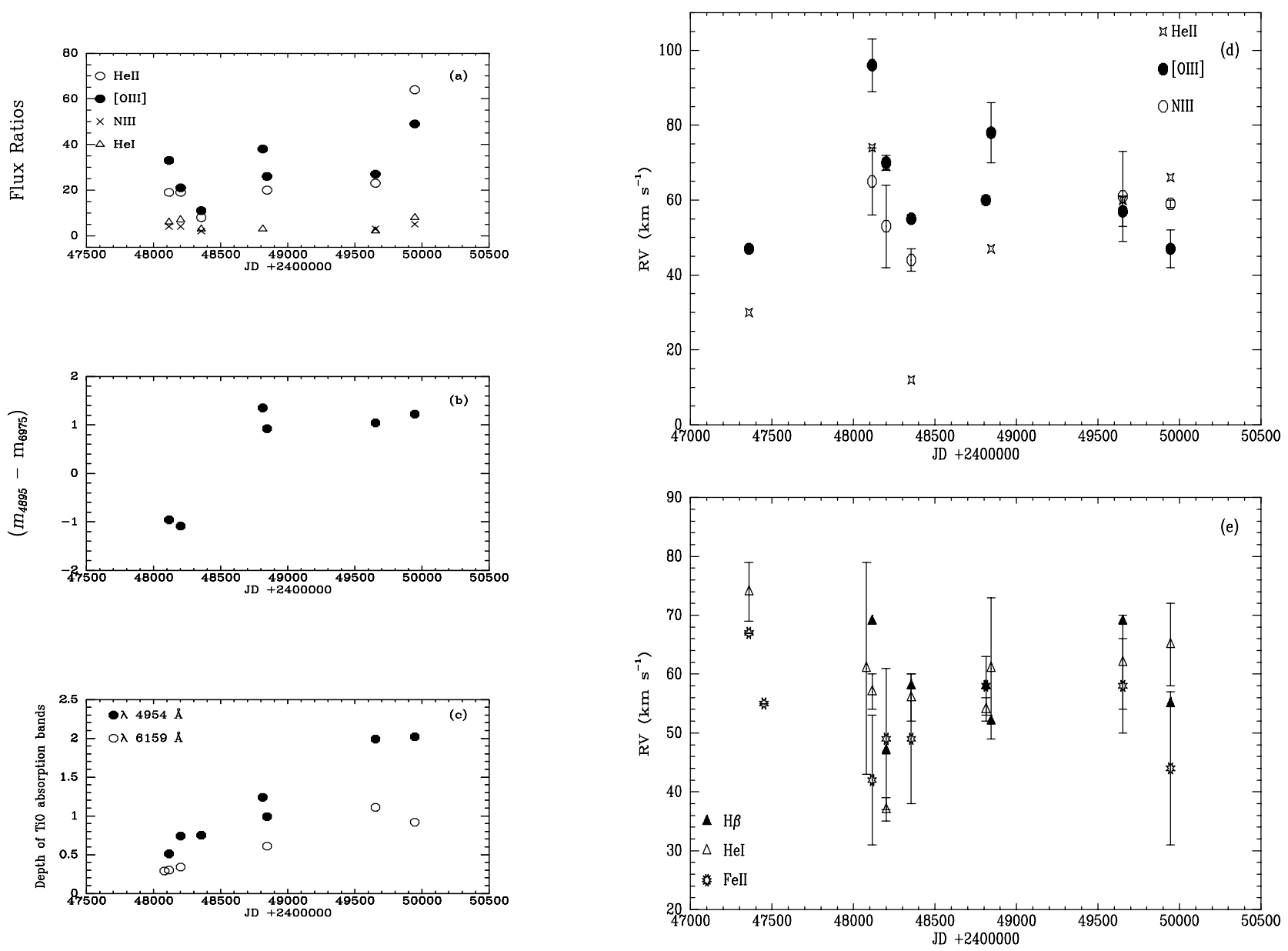

Fig. 2. a) Intensity ratio variations of He I, He II, N III and [O III] observed in Hen 1761. b) "color index" $\left(m_{4895}-m_{6975}\right)$. c) depth of TiO absorption bands at $\lambda 4954 \AA$ and $\lambda 6159 \AA$. d) and e) Radial velocities obtained from different ions. Radial velocity values published by van Winckel et al. (1993), were included in the plots between HJD 2447360 and 2447790

\subsection{Radial velocities}

A complete summary of the heliocentric radial velocities of Hen 1761 are given in Table 3. The individual emission and absorption lines have been measured by fitting a Gauss function to the profiles. The average values of the radial velocities, the corresponding mean errors and the measured line number within brackets are indicated for differents epochs.

The radial velocities have also a variable behavior with time. The Fig. $2 \mathrm{~d}$ shows the radial velocity curves corresponding to the high ionization potential ions, He II and [O III], while the Fig. 2e shows those of the low ionization potential ions H, He I and Fe II separetely. The largest amplitudes of the radial velocity curves and a systematic decrease from August 1990 to April 1991 is observed in the first figure.

Radial velocities of several Balmer lines, $\mathrm{H} \alpha$ to $\mathrm{H} 9$, can be measured in two epochs, 1992 and 1994, except for H8 since it is affected by blending with the He I $\lambda 3888 \AA$. A regression of the Balmer series radial velocities increasing towards the first members of the series may be an evidence that the lines are emitted in an expanding region with a finite optical thickness in these lines.

The singlet and triplet series of the He I lines are shown separately in Table 3 . Their radial velocities show differences which are more noticeable in the Echelle spectra, $17 \mathrm{~km} \mathrm{~s}^{-1}$ in October 1994 and $14 \mathrm{~km} \mathrm{~s}^{-1}$ in August 1995, the triplet emission lines being redshifted with respect to the singlet ones.

In the case of [Fe VI], three double peaked lines $\lambda 4967 \AA, \lambda 4972 \AA$ and $\lambda 5176 \AA$ are measured in August 1995. The mean radial velocities of each peak are $68 \pm$ $8 \mathrm{~km} \mathrm{~s}^{-1}$ and $109 \pm 4 \mathrm{~km} \mathrm{~s}^{-1}$ respectively.

\subsection{Classification of the cool component}

The spectral classification of the cool component was based on the TiO optical molecular-band strengths at $\lambda \lambda$ 6180 and $7100 \AA$ as proposed by Kenyon \& FernándezCastro (1987). We adopted the calibration of the [TiO] 
Table 3. Heliocentric radial velocities of the absorption and emission lines in Hen 1761 in $\mathrm{km} \mathrm{s}^{-1}$

\begin{tabular}{|c|c|c|c|c|c|c|c|c|c|}
\hline \multirow[t]{2}{*}{ Ion } & & \multicolumn{4}{|c|}{ Z-Machine } & \multicolumn{2}{|c|}{ CCD $500 \times 592$} & \multicolumn{2}{|c|}{ CCD $1024 \times 1024$} \\
\hline & & $7 / 90$ & $8 / 90$ & $11 / 90$ & $4 / 91$ & $7 / 92$ & $8 / 92$ & $10 / 94$ & $8 / 95$ \\
\hline$\overline{\mathrm{H} \alpha}$ & $\mathrm{E}$ & 72 & 42 & 58 & & 65 & 63 & 83 & 62 \\
\hline $\mathrm{H} \beta$ & $\mathrm{E}$ & & 69 & 47 & 58 & 58 & 52 & 69 & 55 \\
\hline $\mathrm{H} \gamma$ & $\mathrm{E}$ & & & & & & 54 & 45 & \\
\hline $\mathrm{H} \delta$ & $\mathrm{E}$ & & & & & & 42 & 41 & \\
\hline $\mathrm{H} 8$ & $\mathrm{E}$ & & & & & & 69 & 39 & \\
\hline H9 & $\mathrm{E}$ & & & & & & 39 & 37 & \\
\hline He I $(S)$ & $\mathrm{E}$ & $43(1)$ & $60 \pm 8(3)$ & $35 \pm 1(2)$ & $52 \pm 4(2)$ & $56 \pm 2(3)$ & $49(1)$ & $54 \pm 5(4)$ & $58 \pm 1(4)$ \\
\hline He I $(\mathrm{T})$ & $\mathrm{E}$ & $79(1)$ & $55(1)$ & $38 \pm 7(2)$ & $59(1)$ & $52(1)$ & $73 \pm 6(4)$ & $71 \pm 3(3)$ & $72 \pm 4(3)$ \\
\hline Не ІІ $\lambda 4685$ & $\mathrm{E}$ & & 74 & 69 & 12 & & 47 & 60 & 66 \\
\hline $\mathrm{N}$ III & $\mathrm{E}$ & & $65 \pm 6(2)$ & $53 \pm 8(2)$ & $44 \pm 2(2)$ & & & $61 \pm 5(5)$ & $54 \pm 1$ \\
\hline$[\mathrm{O}$ III $]$ & $\mathrm{E}$ & & $96 \pm 5(2)$ & $70 \pm 1(2)$ & $55 \pm 0(2)$ & $60 \pm 1(2)$ & $78 \pm 6(2)$ & $57 \pm 2(3)$ & $47 \pm 3(3)$ \\
\hline $\mathrm{Na}$ I (D) & A & $64 \pm 5(2)$ & $25: \pm 4(2)$ & $88 \pm 13(2)$ & & & $80 \pm 22(2)$ & $55 \pm 1(2)$ & $59 \pm 0(2)$ \\
\hline$[\mathrm{Ca} \mathrm{v}]$ & $\mathrm{E}$ & & & & & & & $63(1)$ & \\
\hline Fe II & $\mathrm{E}$ & & $42 \pm 4(7)$ & $49 \pm 4(7)$ & $49 \pm 4(7)$ & $58 \pm 3(3)$ & & $59 \pm 3(8)$ & $44 \pm 6(5)$ \\
\hline Ti II & $\mathrm{A}$ & & $65 \pm 4(9)$ & & $72 \pm 9(3)(\mathrm{E})$ & & & $89 \pm 5(12)$ & $56 \pm 4(19)$ \\
\hline$[\mathrm{Fe} \mathrm{VI}]$ & $\mathrm{E}$ & & & & & & & $71 \pm 6(3)$ & $94 \pm 5(3)$ \\
\hline$[\mathrm{Fe}$ VII $]$ & $\mathrm{E}$ & & & & & & & & $62 \pm 7(2)$ \\
\hline
\end{tabular}

Notes:

$(\mathrm{S})=$ singlet; $(\mathrm{T})=$ triplet; $(\mathrm{D})=$ doublet; $\mathrm{E}=$ emission line; $\mathrm{A}=$ absorption line

$(n)=$ number of measured lines

: dubious value.

indices with the spectral types of $\mathrm{M}$ giants given by these authors and both indices, $[\mathrm{TiO}]_{1}$ and $[\mathrm{TiO}]_{2}$, were calculated with the observations of August 1992 and October 1994. The spectral type obtained from the first index $[\mathrm{ST}]_{1}$, is earlier than that given by the second one $[\mathrm{ST}]_{2}$, indicating that the first index is more affected by the contribution of the hot component. In the case of the 19921995 observations, the fluxes were measured separately in each spectra to obtain, for each epoch, the mean indices with their respective internal mean errors.

In Table 4 we can see that the spectral type of the cool star gets increasingly later during the period of our observations. This behavior could be interpreted either as a heating suffered by the giant during the first observations or as the result of variations in the blue continuum. Figure 2c shows variations of the depth of TiO absorption bands in connection with the local continuum level. Two strong absorption bands at $\lambda 4954$ and $\lambda 6159$ have been measured (see Table 4), and we notice that the relative depth of each band increases at the time the spectral type of the giant becomes later, but the amplitude of these variations seems to be more important toward the shorter wavelengths. Besides, if we consider the behavior of the color index $C I=\left(m_{4895}-m_{6975}\right)$ (Fig. $\left.2 b\right)$ we may conclude that the observed changes in the spectral type of the giant are the result of a dilution effect of an additional blue continuum, coming from the hot source and veiling the $\mathrm{M}$ spectrum. The influence of the blue continuum was decreasing in time, as it is shown in the Figs. $2 b$ and c. Consequently we might consider that the spectral type of the cool component in Hen 1761 is as later as M5 III and according to the published near-infrared data from 1972 (Glass \& Webster 1973) and 1990 (Munari et al. 1992), no change is registered in the $I R$ colors, specially in the $K$ and $L$ bands.

\subsection{Reddening and distance}

Before deriving the physical parameters of Hen 1761, it is important to determine the interstellar extinction and distance of the system. We present several ways to determine the reddening, adopting in all the cases, the interstellar extinction curve given by Savage \& Mathis (1997).

The Balmer decrement provides a good reddening diagnostic considering that the optical $\mathrm{H}$ I recombination lines are formed under case B conditions. But this is not the case in the majority of the S-type symbiotic stars, which show significant departures from case B in the lower Balmer series members, due to self-absorption effects. Netzer (1975) has computed the self-absorption effects on hydrogen line intensities in dense nebulae, as functions of Ly- $\alpha$ and $\mathrm{H} \alpha$ optical depths $\left(\tau_{\alpha}, \tau_{\mathrm{H} \alpha}\right)$. By using his results and comparing them with the mean values of our intensity ratios observed in August 1992, October 1994 and August 1995, $I(\mathrm{H} \alpha) / I(\mathrm{H} \beta)=5.4 \pm 1.0 ; I(\mathrm{H} \gamma) / I(\mathrm{H} \beta)=$ $0.37 \pm 0.05 ; I(\mathrm{H} \delta) / I(\mathrm{H} \beta)=0.22 \pm 0.07$; we can derive $E(B-V)=0.26 \pm 0.04$, corresponding to $\tau_{\mathrm{H} \alpha} \sim 20$ and $\tau_{\alpha}=10^{4}$, and densities of $n_{\mathrm{e}}=10^{9} \mathrm{~cm}^{-3}$. The upper Balmer series members have nearly constant intensity 
Table 4. Spectral type of the cool component, color index CI $=\left(m_{4895}-m_{6975}\right)$ and TiO relative depths

\begin{tabular}{|c|c|c|c|c|c|c|c|}
\hline Epoch & {$[\mathrm{TiO}]_{1}$} & {$[\mathrm{ST}]_{1}$} & $\overline{\mathrm{TiO}]_{2}}$ & {$[\mathrm{ST}]_{2}$} & CI & $\lambda 4954$ & $\lambda 6159$ \\
\hline July'90 & 0.29 & M1.0 & & & & & 0.29 \\
\hline August'90 & 0.34 & M1.4 & & & -0.96 & 0.51 & 0.30 \\
\hline November'90 & 0.33 & M1.3 & & & -1.09 & 0.74 & 0.34 \\
\hline April'91 & & & & & & 0.75 & \\
\hline July'92 & & & & & +1.35 & 1.24 & \\
\hline August'92 & $0.52 \pm 0.01$ & M3.1 \pm 0.1 & $0.78 \pm 0.06$ & $\mathrm{M} 4.3 \pm 0.3$ & +0.92 & 0.99 & 0.61 \\
\hline October'94 & $0.47 \pm 0.02$ & $\mathrm{M} 2.6 \pm 0.2$ & $0.95 \pm 0.02$ & $\mathrm{M} 5.1 \pm 0.8$ & +1.04 & 1.99 & 1.11 \\
\hline August'95 & $0.76 \pm 0.01$ & $\mathrm{M} 5.3 \pm 0.1$ & & & +1.34 & 2.02 & 0.92 \\
\hline
\end{tabular}
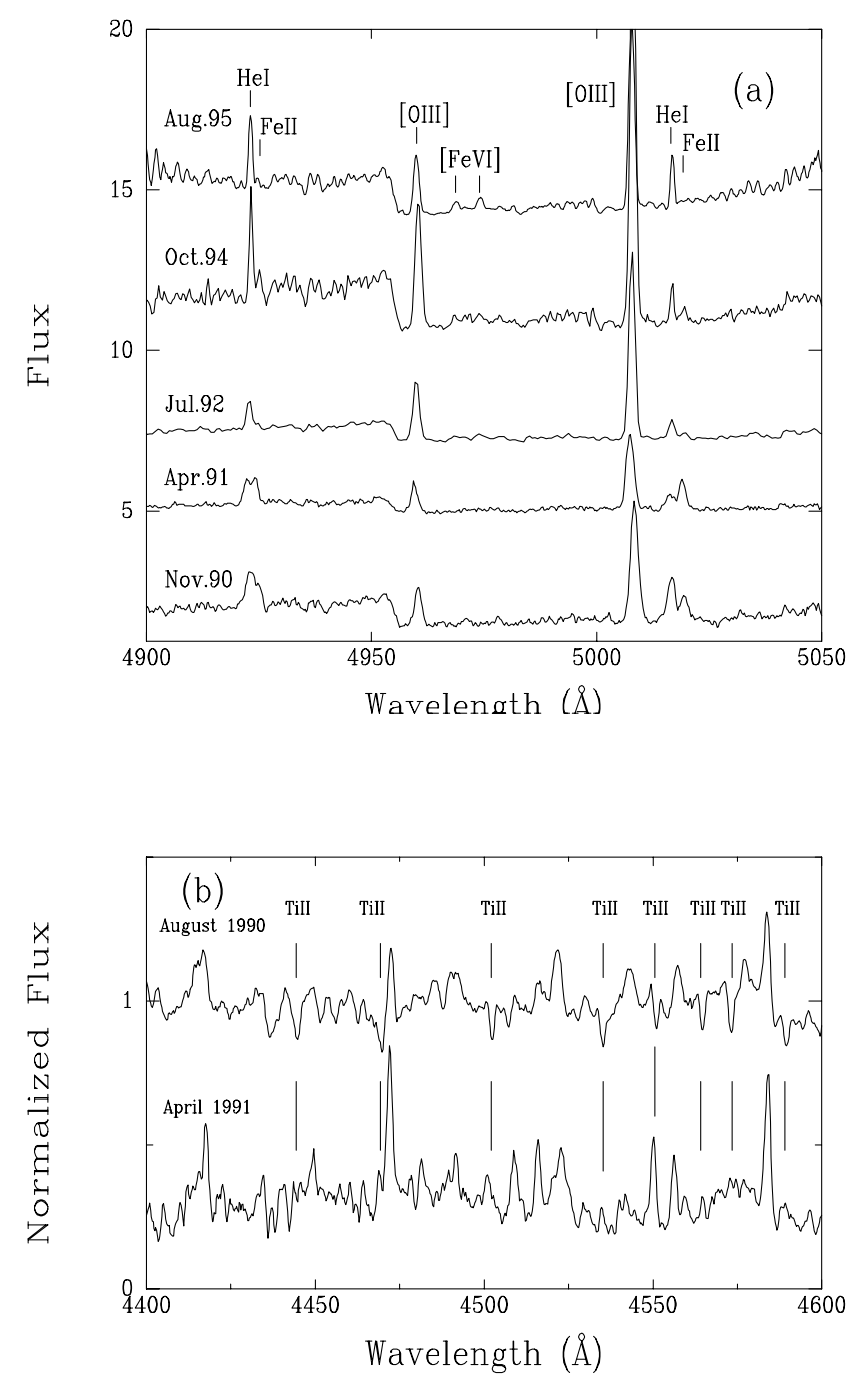

Fig. 3. The spectrograms show intensity variations in the lines of HeI, FeII and TiII. a) In August 1995, FeII is very weak and the spectrum develops forbidden lines of high ionization such as [Fe VI]. b) In August 1990, there is a greater number of absorptions in the blue region, particularly Ti II absorption lines, which disappear in April 1991 or are visible as weak emissions

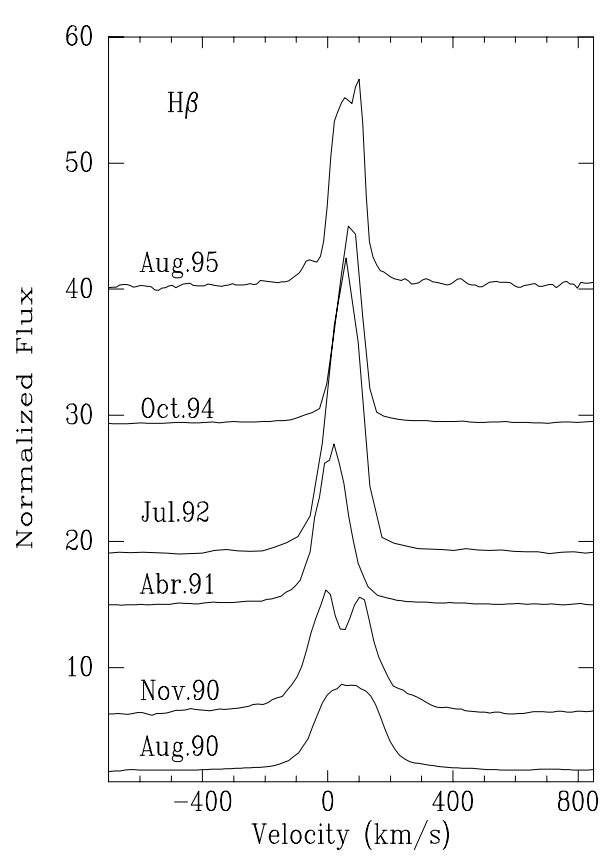

Fig. 4. Variations in the shape of the $\mathrm{H} \beta$ profile along the time. The double-peaked profile observed in November 1990, becomes a single asymmetric emission. Later, in 1995, the $\mathrm{H} \beta$ profile shows an incipient central absorption

ratios in August 1992 and October 1994, with mean values $I(\mathrm{H} \epsilon) / I(\mathrm{H} \beta)=0.13 ; I(\mathrm{H} 9) / I(\mathrm{H} \beta)=0.07 \pm 0.03$ and $I(\mathrm{H} 10) / I(\mathrm{H} \beta)=0.05 \pm 0.02$. The lines showing severe blending were not considered, such as $\mathrm{H} \epsilon$ on the lower resolution spectrum of August 1992, which was blended with [NeIII] and H8 with He I $\lambda 3888 \AA$. In this case a value of $E(B-V)=0.21 \pm 0.12$ is obtained by comparison with theoretical values of case B-recombination taken from Brocklehurst (1971) for $T_{\mathrm{e}}=10000 \mathrm{~K}$ and $n_{\mathrm{e}}=10^{6} \mathrm{~cm}^{-3}$.

Since the near infrared colors of the $\mathrm{M}$ star have remained virtually constant in Hen 1761, reddening migh also be obtained from the $J K$ photometry and the spectral type of the cool component. By combining the observed $(J-K)$ colour index (Munari et al. 1992), with intrinsic colors given by Bessell \& Brett (1988), and considering that the cool component is a normal M5 giant, we obtain $E(B-V)=0.20$. Then a distance of $d \sim 2.2 \mathrm{kpc}$ is 


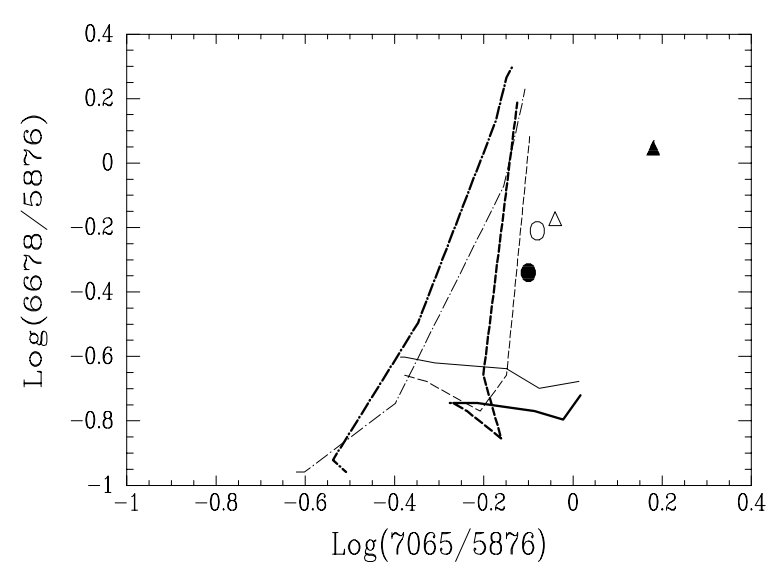

Fig. 5. The He I intensity ratios measured on logarithmic scale in Hen 1761. A comparison with theoretical models taken from Proga et al. is shown for $n_{\mathrm{e}}=10^{12} \mathrm{~cm}^{-3}$ (dot-dashed lines), $n_{\mathrm{e}}=10^{10} \mathrm{~cm}^{-3}$ (dashed lines), $n_{\mathrm{e}}=10^{8} \mathrm{~cm}^{-3}$ (solid lines), $T_{\mathrm{e}}$ of $10000 \mathrm{~K}$ (light lines) and $20000 \mathrm{~K}$ (heavy lines). The observed intensity ratios in Hen 1761 are indicated with symbols for different epochs: 1990 (solid circle), 1992 (open triangle), 1994 (open circle) and 1995 (solid triangle)

obtained by means of the absolute magnitude $M_{K}=$ -6.26 calculated from the $M_{V} \sim-0.3$ (Schmidt-Kaler 1982) and $A_{K}=0.08$.

Whitelock \& Munari (1992) found that many of Stype symbiotics have IR characteristics very similar to those of nonsymbiotics M-giants of the Galactic bulge. Tacking into acount this considerations, a color excess $E(B-V)=0.73$ was obtained by comparing the observed $(J-K)$ colour index with the intrinsic colors given by Frogel \& Whitford (1987) for a bulge M5-giant. Considering the distance modulus to the galactic center, $14.43 \mathrm{mag}$ (Tiede et al. 1995) and the $K$ magnitude given by Frogel \& Whitford (1987), we estimate $M_{K}=-5.2$ and $A_{K}=0.28$. A distance of $d \sim 1.3 \mathrm{kpc}$ is obtained in this case. The reddening resulting when we consider the giant of the Hen 1761 as belonging to the galactic bulge, $E(B-V)=0.73$, conduces to a high reddening, $A_{V}=$ $2.3 \mathrm{mag}$. Consequently the intrinsic colors $(H-K)$ would be correlated with earlier spectral type $\sim$ M0 - M2 (see Figs. 7a and 7b Whitelock \& Munari 1992), and this is inconsistent with our considerations about the spectral type of the cool star. While the reddening estimated for the $\mathrm{HI}$ line emissions is very similar than that derived by comparing the $J-K$ colors observed in Hen 1761 with those for normal giants. This means that the cool component is as reddened as the nebular region of the system. Circumstellar dust around the giant is not important, as usual in S-type symbiotic systems. In this sense, the IRAS data for S-type symbiotic stars (Kenyon et al. 1988), indicated that Hen 1761 has flux ratios, [25/12] 0.2 and $[60 / 25] \sim 1.0$, similar to those of normal M giant stars.
Table 5. Physical parameters of the hot component

\begin{tabular}{lccc}
\hline Epoch & $T_{\mathrm{h}}$ & $L_{\mathrm{h}}$ & $R_{\mathrm{h}}$ \\
& $\mathrm{K}$ & $L_{\odot}$ & $R_{\odot}$ \\
\hline August'90 & 112000 & 90 & 0.02 \\
November'90 & 112000 & 120 & 0.03 \\
April'91 & 90000 & 160 & 0.05 \\
August'92 & 114000 & 200 & 0.04 \\
October'94 & 120000 & 200 & 0.03 \\
August'95 & 160000 & 70 & 0.01 \\
\hline
\end{tabular}

In the next sections we will correct the line fluxes for interstellar reddening adopting the values: $E(B-V)=$ 0.20 and $A_{V}=0.6$.

\subsection{Hot component}

The temperature of the hot component has been estimated from the intensity ratios of the HI, HeI and He II recombination lines under certain assumptions (Kenyon (1986) and references therein). We give in Table 5, the temperatures of the hot component $T_{\mathrm{h}}$ for each epoch of observation, by using Iijima's method and the de-reddened flux ratios of (He II $\lambda 4686) / \mathrm{H} \beta$ and $(\mathrm{He} \mathrm{I} \lambda 4471) / \mathrm{H} \beta$.

The $T_{\mathrm{h}}$ in turn leads to a crude estimation of the blackbody luminosity and radius of the hot star from H I, He I and HeII recombination line fluxes assuming theoretical emissivities of case B (Harman \& Seaton 1966), adopting $T_{\mathrm{e}}=10000 \mathrm{~K}$ and our estimation of distance $d \sim 2.2 \mathrm{kpc}$.

\subsection{Nebulae}

We are constrained to estimate physical conditions in the nebulae of Hen 1761 with the emission lines observed in the optical region. The most popular line ratio that has been used for the determination of $T_{\mathrm{e}}$ and $n_{\mathrm{e}}$ is [O III] $[I(4959)+I(5007)] / I(4363)$. When it was possible to measure the three [O III] emission lines in our spectra, the dereddened intensity ratio increased slightly from 2.2 in August 1992 to 2.5 in October 1994 and to 2.9 in August 1995, which corresponds to $T_{\mathrm{e}}=10000 \mathrm{~K}$, using the Kafatos \& Lynch (1980) diagnostic diagram and the corresponding densities between $n_{\mathrm{e}}=610^{7}-310^{7} \mathrm{~cm}^{-3}$ have been calculated on the basis of Seaton's (1975) relation.

In a large sample of galactic symbiotic stars, Proga et al. (1994) found that, besides distinguishing between S and $\mathrm{D}$ type symbiotics, the He I $\lambda 6678 / \lambda 5876$ ratio provides an useful tool for approximating the physical conditions within the nebulae. The $I(\lambda 6678) / I(\lambda 5876)$ ratio in Hen 1761 is $\gtrsim 0.5$ which corresponds to a S-type symbiotic. Some comments on the position of our object in the $I(\lambda 6678) / I(\lambda 5876)$ vs. $I(\lambda 7065) / I(\lambda 5876)$ diagram may be worthwhile. In Fig. 5 we can see that the positions of the points fall near Proga et al.'s models for $n_{\mathrm{e}} \sim 10^{10} \mathrm{~cm}^{-3}$ and temperature $10000 \mathrm{~K}$. The figure 
shows also that the line ratios appear to be variable, increasing with decreasing $n_{\mathrm{e}}$ since 1990 to 1995 . Proga et al. found that the $I(\lambda 6678) / I(\lambda 5876)$ ratio variations are, in most systems, correlated with $V$, in the sense that lower intensity ratios seem to be associated with optical maxima, either during an eruption when the system changes from quiescence to an outburst stage (i.e. RS Oph) or as a function of the photometric phases along an orbital motion (i.e. V443 Her, AG Peg). It is not clear at the moment which is the case of Hen 1761, and it would be necessary to ascertain the origin of the observed variations first.

The [Fe VII] lines at $\lambda \lambda$ 4942, 4989, 5159, 5721 and $6087 \AA$, are the higher ionization forbidden lines found in Hen 1761 in the spectra of 1995. Nussbaumer \& Storey (1982) give relative intensities of [Fe VII] lines as a function of the electron density and electron temperature in the region where these lines are formed. Our dereddened values of the optical line ratios 5159/6087 and 4942/6087 are 0.63 and 0.20 respectively. The first one is larger than the maximum theoretical values presented by Nussbaumer \& Storey but the second line ratio suggests that the [Fe VII] lines are emitted in a region where the electron temperature is very high, $T_{\mathrm{e}} \gtrsim 60000 \mathrm{~K}$ and the density is in the order of $n_{\mathrm{e}}=10^{7} \mathrm{~cm}^{-3}$.

Some considerations about the observed variations in the physical conditions in the nebulae of Hen 1761 will be made in the next sections.

\section{Discussion}

The timescale of some of the variations is probably very short, one month at least (July and August 1990) according to our observations. Moreover, taking into account Munari's et al. photoelectric observations performed two weeks before our spectroscopic observations of July/90, variations of $\Delta U=0.17 \mathrm{mag}, \Delta B=0.09 \mathrm{mag}, \Delta V=0.05$ mag, $\Delta R=0.02 \mathrm{mag}$ and $\Delta I=0.01 \mathrm{mag}$ occur from one night to the next one, tending to increase the photometric gradient with decreasing wavelength and can thus be associated with the hot component. These rapid photometric variations or "flickering" effect are observed in other symbiotic systems with low hot component luminosities and it is usually interpreted as some measure of fluctuations in the accretion rate (Dobrzycka et al. 1996).

On the other hand, there are variations that seem to take place in Hen 1761 in a longer timescale. [O III] $\lambda 5007 \AA$ was present on the spectra obtained in 1988, it disappeared in 1989 and later since 1990, together with the other high-excitation emission line He II $\lambda 4686 \AA$, displayed important changes in the relative fluxes, reaching a minimun in 1991 and then, increasing until 1995. If we assume that the [O III] lines arise from an homogeneous nebula with $T_{\mathrm{e}}=10000 \mathrm{~K}$ and electron density of $n_{\mathrm{e}}=$ $10^{7}-10^{8} \mathrm{~cm}^{-3}$, the size of the [O III] emitting region can be calculated (Mikolajewska 1985). For a mean unreddened flux of the $\lambda 5007$ line, $F(5007)=1.8510^{-12} \mathrm{erg} \mathrm{cm}^{-2} \mathrm{~s}^{-1}$, and adopting the distance $d=2.2 \mathrm{kpc}$, we obtain a range for the radius of the nebula of 4 to $7 \mathrm{AU}$. If a typical binary separation of $2 \mathrm{AU}$ for S-type symbiotic stars (Kenyon 1986; Schmid 1995) is considered, the [O III] lines are not produced close to the hot component and consequently, their radial velocity variations would hardly correspond to the orbital motion.

When the cool component displays the earlier spectral type, M1.0, an additional blue continuum, coming from the hot source is veiling the $M$ spectrum. In the blue region, metallic lines are in absorption; the forbidden lines are weaker; the high ionization emission lines are absent and the Fe II emission lines are stronger. A considerable weakening in the blue continuum is observed since July 1992. The TiO absorption band depths became greater and consequently the spectral type of the giant gets increasingly later than the former observations. During the latest observations the forbidden lines requiring still higher excitation conditions (e.g. [Fe VI], [Fe VII] and $[\mathrm{CaV}])$ are detected for first time in Hen 1761, some of them with a double-peaked profile as has been observed in other symbiotic stars. The Fe II emission lines show a systematic decrease of intensities and almost disappear in 1995.

Strong central absorptions are visible in the $\mathrm{H}$ lines particularly $\mathrm{H} \alpha$ and $\mathrm{H} \beta$ with variations in time. Many authors have suggested that these profiles are originated in an accretion disc which surrounds the hot component of the system.

The absence of the Raman scattered OVI emission lines $\lambda \lambda 6825,7082 \AA$ deserves to be mentioned. These lines are almost always present in symbiotic systems with high excitation lines, such as [Fe VII] or [Ne V] (Schmid 1996) but according to Kenyon (1986) the symbiotics that have noticeable [Fe VII] emissions and no trace of $\lambda \lambda 6825$, $7082 \AA$, are likely candidates for an accreting main sequence star as the hot source.

\section{Conclusions}

We summarize our study by saying that Hen 1761 is an S-type symbiotic star with important spectral variations. Some of the spectral changes can be explained if we consider that Hen 1761 has to go through outburst and quiescence stages, in the case that the system contains a red giant star M5 and a hot white dwarf with an accretion disk, which powers the activity of the system. Unfortunately, it is not possible to specify both stages due to the lack of a light curve following the spectral changes. During the activity in 1990-91 the spectrum shows the characteristic outburst properties as is somtimes observed in symbiotic stars: the presence of $\mathrm{HI}$, He I, He II, N III and Fe II emission lines and forbidden lines of [Fe II], [Ne III] and [O III]. Absorption lines like the doublet $\mathrm{Na}$ I and $\mathrm{Ca}$ I $\lambda 4226 \AA$ 
are also present in this phase. On the other hand, a blue continuum veils the giant's features and an earlier spectral type for the cool component is inferred from the optical spectrum. The quiescence phase may have started in 1992, when the Fe II emission lines decreased, the forbidden lines of low-excitation [Fe II] and [Ne III] disappeared and forbidden lines requiring still higher excitation conditions (e.g. [Fe VI], [Fe VII] and [Ca V] ) are observed. At the same time, the veiling of the absorption bands on the blue continuum decreases substantially. The [O III] emission lines are present as much as the He II ones, during both phases but their intensities are increasingly noticeable on our quiescent observations. The variability of the He I intensity ratios presented in Fig. 5 suggests that the outburst produces changes in the density of the nebula where these lines are formed.

Other spectral variations are observed in Hen 1761 due perhaps to orbital motion or associated with the flickering activity. In order to separate the causes of the variations, a series of photometric and spectroscopic observations are necessary. Thus, it would be very important to analyse the UV spectrum of Hen 1761 with the aim of learning more about the nature of the hot component and confirming the existence of an accretion disk.

Acknowledgements. The authors wish to thank Prof. J. Sahade for careful reading of the manuscript. The authors also acknowledge the use of the CCD and data acquisition system supported under U.S. National Science Foundation grant AST90-15827 to R.M. Rich.

\section{References}

Allen D.A., 1980, MNRAS 192, 521

Allen D.A., 1982, in The Nature of Symbiotic Stars, IAU Coll. 70, Friedjung M., Viotti R., (eds.). Reidel, Dordrecht, p. 27
Allen D.A., 1984, Proc. Astron. Soc. Aust. 5, 367

Baldwin J.A., Stone R.P.S., 1984, MNRAS 206, 241

Barbá R., Brandi E., García L., Ferrer O., 1992, PASP 104, 33

Bessell M.S., Brett J.M., 1988, PASP 100, 1134

Brocklehurst M., 1971, MNRAS 153, 471

Dobrzycka D., Kenyon S.J., Milone A.A., 1996, AJ 111, 414

Frogel J.A., Whitford A.E., 1987, ApJ 320, 199

Glass I.S., Webster B.L., 1973, MNRAS 165, 77

Harman R.J., Seaton M.J., 1966, MNRAS 132, 15

Kafatos M., Lynch J.P., 1980, ApJS 42, 611

Kenyon S.J., 1986, The Symbiotic Stars. Cambridge Univ. Press. Cambridge

Kenyon S.J., Fernández Castro T., 1987, AJ 93, 938

Kenyon S.J., Fernández Castro T., Stencel R.E., 1988, AJ 95, 1817

Lund L., Tuvikene T., 1987, Afz 26, 457

Mayall M., 1951, Bull. Harvard College Obs. 920, 32

Mikolajewska J., 1985, Acta Astron. 35, Nos. 1-2

Munari U., Yudin B.F., Taranova O.G., et al., 1992, A\&AS 93, 383

Netzer H., 1975, MNRAS 171, 395

Nussbaumer H., Storey P.J., 1982, A\&A 113, 21

Proga D., Mikolajewska J., Kenyon S.J., 1994, MNRAS 268, 213

Savage B.D., Mathis J.S., 1979, ARA\&A 17, 73

Schmid H.M., 1989, A\&A 211, L31

Schmid H.M., 1995, MNRAS 275, 227

Schmid H.M., 1996, MNRAS 282, 511

Schmidt-Kaler Th., 1982, in Landolt-Börnstein, New Series. Berlin Springer Vol. VI/2, p. 18

Schulte-Ladbeck R.E., Aspin C., Magalhães A.M., Schwarz H.E., 1990, A\&AS 86, 227

Seaton M.J., 1975, MNRAS 170, 475

Stone R.P.S., Baldwin J.A., 1983, MNRAS 204, 347

Thackeray A.D., 1954, Observatory 74, 258

Tiede G.P., Frogel J.A., Terndrup D.M., 1995, AJ 110, 2788

van Winckel H., Duerbeck H., Schwarz E., 1993, A\&AS 102, 2

Whitelock P.A., Munari U., 1992, A\&A 255, 171

Wright A.E., Allen D.A., 1978, MNRAS 184, 893 